\title{
SOLUBLE GROUPS WITH COMPLEMENTED SUBNORMAL SUBGROUPS
}

\author{
R. KOCHENDÖRFFER \\ (Received 19 March 1969) \\ To Bernhard Hermann Neumann on his 60th birthday \\ Communicated by G. B. Preston
}

A subgroup $A$ of a group $G$ is said to be complemented in $G$ if $G$ contains a subgroup $C$ such that

$$
G=A C, A \cap C=1 .
$$

Every subgroup $C$ with this property is called a complement of $A$ in $G$. Various results have been obtained about groups in which each member of a given set of subgroups is complemented. Some of these results state, roughly speaking, that the existence of complements of all members of a given set of subgroups implies that all members of a larger set are also complemented. In this paper we derive another theorem of this kind.

Adopting a notation introduced by $\mathrm{C}$. Christensen $[1]$ we define a group $G$ to be an $a C$-group ( $s C$-group, $n C$-group) if every subgroup (every subnormal subgroup, every normal subgroup) of $G$ is complemented in $G$. We are concerned with conditions under which an $s C$-group is an $a C$-group. This is certainly not always the case since every $a C$-group is soluble (cf. [2] for finite groups and [3], [4] for arbitrary groups). On the other hand, not all $s C$-groups are soluble as any non-abelian simple group shows. Therefore it is natural to restrict oneself to soluble groups. We shall prove the following:

THEOREM. Every soluble $s C$-group is an aC-group.

We shall need the following lemmas:

Lemma 1. Every subnormal subgroup of an $s C$-group is an $s C$-group.

Since subnormality is transitive the proof is simple and will be omitted.

Lemma 2 (cf. [5], Lemma 4). If $B \leqq A \leqq G$, if $A$ and $B$ are complemented in $G$, and if $N$ is a complement of $B$ in $G$, then $A$ has a complement in $G$ which is contained in $N$.

For the proof we refer to [5]. 
Lemma 3. Let $G$ be an $s C$-group and $A$ a normal subgroup of $G$. Then every complement of $A$ in $G$ is an $s C$-group.

Proof. Let $B$ be any complement of $A$ in $G$. For a subnormal subgroup $S$ of $B$ the product $A S$ is subnormal in $G$. Therefore there exists a subgroup $C$ of $G$ such that $A S C=G, A S \cap C=1$. By Lemma 2 we may assume that $C \leqq B$. This gives $S C=B, S \cap C=1$ which completes the proof.

Lemma 4 (cf. [4], Theorem 7, Corollary 2). Let $A$ be an abelian normal subgroup of $G$ and let $A$ be a (restricted) direct product of subgroups whose orders are prime numbers. If every subgroup of $A$ is complemented in $G$, then there exists a direct decomposition of $A$ into groups of prime orders which are all normal in $G$.

Proof. By Zorn's Lemma there exists a maximal set of independent subgroups of prime orders of $A$ which are normal in $G$. Let $M$ be the direct product of all members of such a maximal set. Let us assume that $M$ is a proper subgroup of $A$.

Then $A$ has a direct decomposition $A=M \times A_{1}$. Since $A_{1} \neq 1$ there is a subgroup $P$ of prime order such that $A_{1}=P \times A_{2}$ where the case $A_{2}=1$ is not excluded. We write $A=N \times P$ with $N=M \times A_{2}$. By the condition on $G$ there exists a subgroup $H$ of $G$ such that

$$
G=N H, N \cap H=1 \text {. }
$$

This implies $A=N \times Q$ where $Q=A \cap H$. Since $A$ is normal in $G$ it follows that $Q$ is normal in $H$, and since $A$ is abelian, $Q$ and $N$ commute elementwise. Making use of (1) we conclude that $Q$ is normal in $G$. Moreover $P \cong A / N \cong Q$. Thus $Q$ is a subgroup of $A$ of prime order which is independent of $M$ and normal in $G$. This contradicts the assumption on $M$ so that the Lemma is proved.

In view of Lemma 4 a direct decomposition of $A$ will be said to be normal in $G$ if the direct factors have prime orders and are normal in $G$.

Proof of The THeOREm. Let $G$ be a soluble $s C$-group and let

$$
G=G^{(0)}>G^{(1)}>G^{(2)}>\cdots>G^{(n)}=1
$$

be its derived series. Since $G$ is in particular an $n C$-group we can apply Lemma (4.3) of $[1]$ to obtain a factorization

$$
G=A_{1} A_{2} \cdots A_{n}
$$

where $A_{1}, A_{2}, \cdots, A_{n}$ are abelian subgroups of $G$ which satisfy the following conditions
(i) $A_{i} \cap A_{i+1} A_{i+2} \cdots A_{n}=1 \quad(i=1, \cdots, n-1)$
(ii) $A_{i+r} \leqq N_{G}\left(A_{i}\right)$ $(i=1, \cdots, n-1 ; r=1, \cdots, n-i)$
(iii) $G^{(n-i)}=A_{1} \cdots A_{i}$ $(i=1, \cdots, n)$. 
By Lemma 1, every group $G^{(n-i)}$ is an $s C$-group and hence it follows from Lemma 3 that every $A_{i}$ is an $s C$-group. This means that every subgroup of the abelian group $A_{i}$ is a direct factor. As is well-known $A_{i}$ is therefore a direct product of subgroups whose orders are prime numbers.

For every $i, 1 \leqq i \leqq n-1$,

$$
G_{i}=A_{i} \cdots A_{n}
$$

is an $s C$-group by Lemma 3 . Every subgroup of $A_{i}$ is subnormal in $G_{i}$ and is therefore complemented in $G_{i}$. Hence, by Lemma 4 , every $A_{i}$ has a direct decomposition which is normal in $G_{i}$.

We choose arbitrary elements $u, v, w, x$ of $G$. Corresponding to (2) we have

$$
u=u_{1} u_{2} \cdots u_{n}, u_{i} \in A_{i} .
$$

For each $u_{i}, i=1, \cdots, n$, we form the direct product $U_{i}$ of all those simple direct factors of a normal decomposition of $A_{i}$ which contain components $\neq 1$ of $u_{i}$. In an analogous way we define the subgroups $V_{i}, W_{i}, X_{i}$ of $A_{i}$. It follows that for every $i, 1 \leqq i \leqq n$,

$$
R_{i}=U_{i} V_{i} W_{i} X_{i}
$$

is a finite subgroup of $A_{i}$ which is normal in $G_{i}$ and hence, in particular, $R_{i} R_{j}=R_{j} R_{i}$. Therefore

$$
R=R_{1} R_{2} \cdots R_{n}
$$

is a finite subgroup of $G$ which contains $u, v, w, x$. Since each $R_{i}$ is a product of factors of a normal decomposition of $A_{i}$ it is easy to see that $R$ is supersoluble. Hence, by a well-known theorem (cf. [6], Theorem 7.2.13) the derived group $R^{\prime}$ is nilpotent.

Let $S$ be a Sylow $p$-subgroup of $R$. From the construction of $R$ it is evident that $S$ is obtained by forming consecutive splitting extensions of finite elementary abelian $p$-groups by finite elementary abelian $p$-groups. Moreover each Sylow $p$-subgroup of $R_{i}$ is a product of factors of a normal decomposition of $A_{i}$. Hence $S$ is abelian. It follows that the Sylow subgroups of the nilpotent group $R^{\prime}$ are abelian so that $R^{\prime}$ itself is abelian, i.e. $R^{\prime \prime}=1$. This gives $[[u, v],[w, x]]=1$ and since $u, v, w, x$ are arbitrary elements, we obtain the result that $G^{\prime \prime}=1$.

Thus the structure of $G$ can be described as follows. $G$ is a splitting extension of an abelian group $A\left(=G^{\prime}\right)$ by an abelian group $B$ where both $A$ and $B$ are direct products of groups whose orders are prime numbers. Moreover, $A$ has a direct decomposition which is normal in $G$.

From results of N. V. Baeva [3] and S. N. Cernikov [4] it now follows that $G$ is an $a C$-group. We repeat the simple proof. For any subgroup $T$ of $G$ let $A_{0}=A \cap T$ and let $B_{0}$ be the subgroup of $B$ generated by the 
$B$-components of the elements of $T$. We can find a complement $\bar{A}_{\mathbf{0}}$ of $A_{0}$ in $A$ which is the product of suitable direct factors of a normal decomposition of $A$ so that $\bar{A}_{0} \triangleleft G$. Moreover let $\bar{B}_{0}$ be any complement of $B_{0}$ in $B$. Then $\bar{A}_{0} \bar{B}_{0}$ is a complement of $T$ in $G$.

\section{References}

[1] C. Christensen, 'Complementation in Groups', Math. Z. 84 (1964), 52--69.

[2] P. Hall, 'Complemented Groups', J. London Math. Soc. 12 (1937), $201-204$.

[3] N. V. Baeva, 'Completely Factorisable Groups', Doklady Akad. Nauk S.S.S.R. (N.S.) 93 (1953), 877- -880 .

[4] S. N. Cernikov, 'Groups with Systems of Complemented Subgroups', Mat. Sbornik 35 (77) (1954), $93 \ldots 128$.

[5] N. T. Dinerstein, 'Finiteness Conditions in Groups with Systems of Complemented Subgroups', Math. $Z, 106$ (1968), $321-326$.

[6] W. Scott, Group Theory, Englewood Cliffs, New Jersey: Prentice Hall Inc. 1964.

Department of Mathematics

University of Tasmania

Hobart, Tasmania 